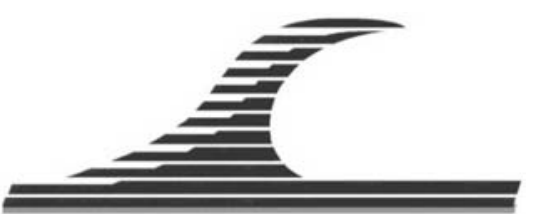

Revue Paralia, Volume 2 (2009) pp s2.1-s2.15

Mots-clés : Sédiments marins contaminés, Traitement par liant

hydraulique, Stabilisation/Solidification, Résistance à la

compression, Projet SEDI.MAR.D. 83.

\title{
Approche économique et validation de méthodes de traitements aux liants hydrauliques de sédiments marins contaminés
}

\author{
Laurent SANNIER ${ }^{1}$, Daniel LEVACHER ${ }^{2}$, Mickaël JOURDAN ${ }^{1}$ \\ ${ }^{1}$ Conseil Général du Var, Direction des Ports, Division Travaux Neufs \\ 390, avenue des Lices - 83076 TOULON Cedex, France. \\ lsannier@cg83.fr;mjourdan@cg83.fr \\ ${ }^{2}$ Université de Caen, Faculté des Sciences, UMR 6143 CNRS - M2C, \\ Esplanade de la Paix, 14032 Caen, France. \\ daniel.levacher@unicaen.fr
}

\section{Résumé :}

Pour une meilleure gestion à terre des sédiments contaminés, considérés comme déchets, une unité pilote de traitements a été mise en œuvre sur un site expérimental, proche de Toulon. Un large éventail de sédiments portuaires représentatif de la variabilité de ces déchets a été acheminé sur cette plate-forme pour y être traité et valorisé et ce, de manière opérationnelle dans le cadre du projet SEDI.MAR.D. 83. Un des objectifs du projet a concerné la valorisation des sédiments à l'aide de liants hydrauliques. Pour ce faire, des essais de faisabilité de stabilisation/solidification sur des sédiments pré traités ou directement traités, ont été entrepris tant du point de vue mécanique qu'environnemental. L'analyse des nombreux résultats d'essais réalisés sur différents sédiments portuaires a permis d'étudier la faisabilité technique de ce type de procédé de traitement par liants, d'optimiser les dosages et les additifs à mettre en œuvre et de confirmer l'acceptabilité environnementale par rapport aux filières de valorisation envisageables pour ces déchets comme matériaux routiers et de remblais ouvrages en terre, talus paysagers, remblaiement de carrière, remplissage de cavités souterraines-. Les possibilités d'emploi d'un tel procédé de traitement par liants hydrauliques sont discutées dans cet article et notamment la discrimination économique des traitements effectués en amont qui permettent d'améliorer la qualité du sédiment pré traité avant stabilisation/solidification par liant. Cet article fait partie d'un ensemble de contributions relatives au projet SEDI.MAR.D. 83, (AQUA et al.., 2009 ; BENOITBONNEMASON et al., 2009 ; LEVACHER et al., 2009 ; SEBY et al., 2009).

Soumis le 15 décembre 2008, accepté le 15 juin 2009, en ligne le 7 septembre 2009. La seule version examinée est celle écrite en français. La ou les autres versions n'étant pas examinées par le comité de rédaction de la revue, sont donc publiées sous l'entière responsabilité du ou des auteurs.

A TRANSLATED VERSION IN ENGLISH IS AVAILABLE ONLINE

Pour citer cet article :

SANNIER L., LEVACHER D., JOURDAN M. (2009). Approche économique et validation de méthodes de traitements aux liants hydrauliques de sédiments marins contaminés. Revue Paralia, $\mathrm{n}^{\circ} 2$, pp s2.1s2.15.

DOI: 10.5150/revue-paralia.2009.s02

(disponible en ligne - http://www.paralia.fr - available online) 


\section{Introduction}

En présence de sédiments plus ou moins contaminés, par des métaux lourds, HAP, TBT, matières organiques, (SANNIER, 2008), la gestion des dragages représente dès lors une problématique économique et technique pour le développement et le maintien des activités portuaires. Face à une situation de blocage de gestion de ces sédiments contaminés qui ne peuvent pas être clapés en mer, des gestionnaires de ports assistés par des bureaux d'études spécialisés ont initié soit des programmes de recherche soit des projets orientés vers une logique opérationnelle pour essayer d’identifier des perspectives possibles de solutions d'emploi à terre de ce nouveau déchet que constituent les sédiments marins contaminés. Dans cette logique, le Conseil Général du Var a initié en 2002 une approche opérationnelle multi partenariale dans le cadre d'un programme spécifique appelé SEDI.MAR.D. 83 (GROSDEMANGE et al., 2008 ; AQUA et al., 2009). Dans cette opération, un des principaux objectifs était d'effectuer des essais de traitements par inertage des contaminants contenus dans les sédiments acheminés sur le site de l'opération en formant des mélanges à base de liants hydrauliques. Le domaine de valorisation des produits ainsi élaborés concerne en priorité les techniques routières (sous couches, remblais). Ainsi, de multiples essais ont été réalisés sur les sédiments provenant de différents ports pour vérifier la faisabilité technique et environnementale des procédés d'inertage à base de ciment comme liant hydraulique principal (BOUTOUIL, 1998 ; COLIN, 2003 ; DUBOIS, 2006 ; SEMCHA, 2006 ; DUAN, 2008 ; TRAN, 2009).

Rappelons que toute application d'une méthode de stabilisation/solidification par traitement à base de liant hydraulique, à des sédiments contaminés se doit de considérer trois aspects, à savoir : un volet technique (comportement mécanique), un volet environnemental (acceptabilité vis-à-vis des normes en vigueur, ADEME et al., 2006) et une nécessaire approche économique (coûts et compétitivité des produits élaborés).

Cet article concerne précisément l'approche économique à travers l'attribution d'une note globale faite à chaque chaîne de traitement en relation avec la formulation (dosages en liants) et l'indicateur mécanique (résistance à la compression simple à 28 jours, notée Rc28, un des critères de base pour un réemploi en techniques routières) retenus. Le volet environnemental tout aussi important dans l'acceptabilité de la valorisation des sédiments contaminés du point de vue sociétal que l'aspect mécanique, n'est pas traité dans cet article. En effet cet article fait partie d'un ensemble de contributions relatives au projet SEDI.MAR.D. 83 dont certaines abordent en détails ce volet sur la même problématique de traitements de sédiments méditerranéens, (BENOIT-BONNEMASON et al., 2009 ; SANNIER, 2008 ; SEBY et al., 2009).

La particularité de ces mélanges sédiment-ciment réside dans le fait que le dit sédiment a subi un ou plusieurs prétraitements qui sont rappelés par la suite avant la description des mélanges (dosages en ciment et additifs). Des mélanges sur des sédiments bruts ont aussi été entrepris de manière à servir de référence. Ainsi un vaste programme 
Approche économique et validation de méthodes de traitements aux liants hydrauliques de sédiments marins contaminés: s2.3

expérimental a d'abord été mené, combinant essais mécaniques et environnementaux (SANNIER, 2008) pour diverses formulations en liants et différents prétraitements appliqués aux sédiments. Ces combinaisons ont servi par la suite de support à l'approche économique proposée. Celle-ci est basée sur l'attribution d'une note économique à la fois aux formulations et aux chaînes de traitement. S'ensuit une analyse de ces évaluations économiques qui permet finalement de discriminer économiquement les traitements effectués en amont permettant d'améliorer la qualité du procédé par liant hydraulique.

\section{Etude expérimentale}

\subsection{Objet de l'étude pilote SEDI.MAR.D. 83}

Cette étude a été élaborée dans le cadre d'un programme de recherche et développement dénommé projet SEDIMARD 83 visant à répondre aux difficultés des maîtres d'ouvrage pour la gestion à terre des sédiments portuaires non immergeables, avec un gisement français estimé pour cette catégorie de sédiments à 10 millions de m3 par an. Le Conseil Général du Var s’est ainsi positionné comme fédérateur pour la recherche de solutions communes. Ce travail expérimental est destiné à tester la faisabilité technique et environnementale de traitements dans le but d'une stabilisation des contaminants présents dans les sédiments marins. Ces procédés conduisent à obtenir un matériau contrôlé qui pourra être valorisé selon ces propriétés dans les domaines du génie civil et de la construction comme granulats et/ou matériau de construction mais encore dans le domaine de l'agriculture comme apport dans la composition des sols, (LEVACHER et al., 2008a, LEVACHER et al., 2008b, LEVACHER et al., 2008c).

L’origine des sédiments dragués provient de dix sites indépendants mais complémentaires afin d'être représentatif de la variabilité de ce déchet et de tester la robustesse de chaque procédé de valorisation.

\subsection{Liste des prétraitements effectués}

Les dix sédiments portuaires prélevés dans le cadre de ce programme ont fait l’objet de divers traitements préalables à l'ajout de liant et ce, individuellement. Ainsi après réception sur le site de l'opération, huit opérations de prétraitement ont été réalisées soit de manière indépendante soit à la suite des unes des autres. Lors de l'arrivée sur le site, les sédiments subissent un tri et une coupure granulométrique et de facto, deux classes de sédiments sont ainsi obtenues : bruts (tri des macro déchets par tamis à $5 \mathrm{~mm}$ ) et dessablés (tri identique au précédent plus hydrocyclonage à $63 \mu \mathrm{m}$ ) qui contiennent les particules les plus fines. Les autres opérations s’appliquent ensuite à ces deux catégories de sédiments. Elles seront alors effectuées soit seule de manière unique soit associées entre-elles, constituant une chaîne de prétraitement amont avant de subir le traitement 
par liant hydraulique, voir tableau I. Dans ce programme, ont ainsi été menées soit une opération relative à :

- une déshydratation qui s’effectue par filtre pressage mécanique dont l'objet est la réduction de la teneur en eau et par conséquence des volumes à traiter,

- un compostage sur une durée de quatre mois afin de réduire la quantité de matière organique,

- une phosphatation caractérisée par un ajout d'acide phosphorique à un dosage d'environ 5\%, pour rendre inerte les contaminants métalliques, (NGUYEN, 2008),

- un chaulage constitué d'un ajout de $20 \%$ de chaux vive dans le but de détruire une partie de la matière organique et d'abaisser la teneur en eau, (REY, 1999),

- une correction granulométrique avec un apport d'un matériau de carrière i.e. un sable fin de même granulométrie. Les pourcentages de sable retenus étaient respectivement de $70 \%$ et $85 \%$ pour $30 \%$ et $15 \%$ de sédiment à traiter. Cette opération permet d'améliorer les caractéristiques mécaniques du matériau ainsi élaboré, (COLIN, 2003 ; DUBOIS, 2006 ; TRAN, 2009),

- une calcination à une température de $450^{\circ} \mathrm{C}$ afin de détruire la matière organique et certains contaminants, (NGUYEN, 2008).

Dans ces prétraitements, aucune opération de broyage ou d'attrition n'a été effectuée sur les sédiments. Même si l'attrition, technique minéralurgique, a montré un intérêt pour la dépollution de sédiments pluviaux (PETAVY et al., 2007 ; PETAVY et al., 2008). Le tableau 1 regroupe les différentes chaînes de traitement que le sédiment a subi (prétraitement) et leurs numéros d'échantillon. Dans le cadre des travaux de recherche, les sédiments présentés ci-dessous ont tous été caractérisés du point de vue chimique avant incorporation de ciment. Il a été constaté qu'aucun des sédiments quelle que soit sa classe, et quels que soient les prétraitements opérés avant incorporation de liants, n'était valorisable, (SANNIER, 2008). En effet, aucun d'entre eux, ne répondait ni aux critères d'acceptabilité proposés pour une valorisation en matériaux routiers (ADEME et al., 2006) ni aux valeurs seuils d'admission en centres de stockage de déchets selon la directive décharge pour déchets (JOCE, 2003). Par ailleurs, ceux-ci ont montré une grande diversité des formes chimiques sous lesquelles se trouvent les métaux, (BENOIT-BONNEMASON et al., 2009 ; SEBY et al., 2009). Cependant, il n'est pas exclu que des comportements différents puissent être observés lors d'un traitement par liant lié à la grande variabilité des états et niveaux de contaminations des sédiments prétraités. 
Approche économique et validation de méthodes de traitements aux liants hydrauliques de sédiments marins contaminés: s2.5

Tableau 1. Liste des procédés de traitements et leurs abréviations.

\begin{tabular}{|c|c|c|}
\hline Abréviations & Chronologie des traitements & $\begin{array}{c}\text { Echantillon } \\
\text { Numéro }\end{array}$ \\
\hline DDCA & DEESSABLÉ - DÉSSHYDRATÉ - CALCINÉ $450^{\circ} \mathrm{C}$ & 2 \\
\hline DD & DEESSABLÉ - DÉSHYDRATÉ & 6 \\
\hline DCO & DESSABLÉ - $\underline{\text { COMPOSTÉ }}$ & 11 \\
\hline BCA & BRUT - CALCINÉ $450^{\circ} \mathrm{C}$ & 15 \\
\hline BCO & BRUT - $\underline{\text { COMPOSTÉ }}$ & 19 \\
\hline $\mathbf{B}$ & BRUT & 21 \\
\hline BPCO & 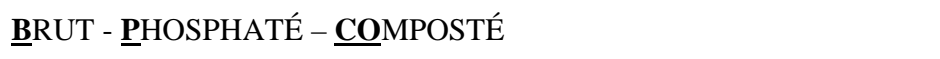 & 24 \\
\hline DCH & DESSABLÉ - $\underline{\text { CHAULÉ }}$ & 27 \\
\hline ВCH & BRUT - $\underline{\text { CHAULÉ }}$ & 29 \\
\hline BCO-PD & 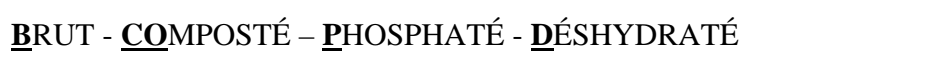 & 34 \\
\hline BCO & BRUT - COMPOSTÉ & \multirow{2}{*}{35} \\
\hline CG (MA) & 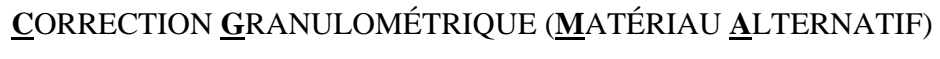 & \\
\hline BCO-PD & 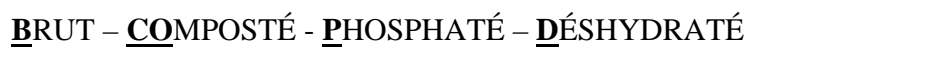 & \multirow{2}{*}{36} \\
\hline CG (MA) & 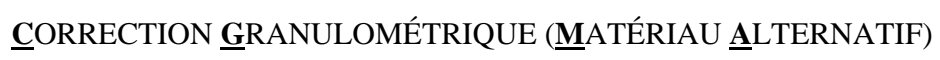 & \\
\hline DCO-P & 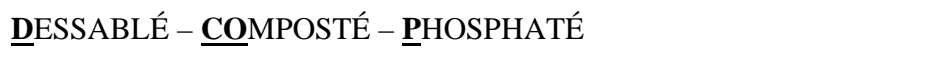 & 41 \\
\hline BPCO & 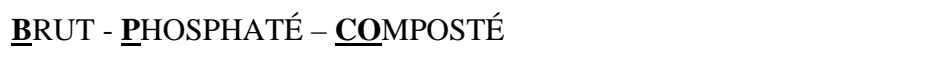 & \multirow{2}{*}{42} \\
\hline CG (MA) & 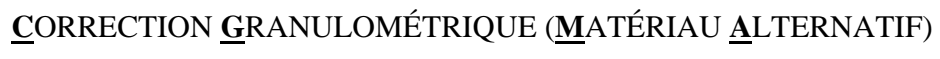 & \\
\hline DPCO & DESSABLÉ - PHOSPHATÉ - $\underline{\text { COMPOSTÉ }}$ & 43 \\
\hline
\end{tabular}

\subsection{Méthodes}

Le traitement des sédiments par liant hydraulique est préparé suivant deux principaux mélanges, à savoir : les mélanges "sédiment et ciment » et les mélanges « sédiment, ciment et additif ». Pour ces dernières formulations, l’objectif est d'étudier l'influence de la présence d'une certaine quantité d'additif réagissant avec les constituants du sédiment.

Les constituants des mélanges sont introduits dans le malaxeur dans l'ordre suivant : vase, additif s'il y a lieu, puis ciment de façon à respecter la cinétique des réactions en présence. Le ciment utilisé est un ciment de type CEM I PM de classe 52,5 $(\mathrm{CaO}=65,3 \%)$. Les additifs sont au nombre de deux et, sont soit de la chaux vive $(\mathrm{CaO}=92 \%)$ pour son action éventuelle sur les matières organiques, et incidemment sur l'amélioration de la résistance à la compression ; soit de la Soproline ${ }^{\circledR}$ (cendre volante à $32.3 \%$ de $\mathrm{CaO}$ ) pour améliorer la réaction pouzzolanique à plus long terme, en permettant notamment aux particules des cendres volantes d'être absorbées par celles du ciment et d'empêcher celui-ci de floculer. Les formulations établies dans ce programme sont rapportées dans le tableau 2. Les proportions envisagées tiennent compte de deux 
composantes : une composante économique limitant la quantité de liant utilisé, et une mécanique liée à la résistance à la compression simple qui est fonction de la proportion de ciment. Un seuil de $1 \mathrm{MPa}$ dit de référence a été retenu compte tenu des valorisations envisagées pour le produit élaboré, i.e. un matériau pelletable, voire un matériau de sous couche routière. Une fois la mise en œuvre terminée, le matériau est incorporé dans des moules cylindriques de diamètre $6 \mathrm{~cm}$ et de hauteur $12 \mathrm{~cm}$ de façon manuelle en trois fois, à chaque remplissage l'éprouvette est tassée manuellement par 3 chocs successifs appliqués verticalement sur le sol. La durée de stockage à température ambiante est de 28 jours.

Tout d'abord, une première série, référencée (A), de mélanges " sédiment-ciment» a été réalisée pour des teneurs pondérales en ciment de $0.5,1,1.25$ et $1.5 \mathrm{~kg}$ pour $10 \mathrm{~kg}$ de sédiment sec. Puis ont été préparés, des mélanges " sédiment-ciment-additifs » qui ont constitué une deuxième série, référencée (B), pour laquelle on a incorporé des additifs (chaux et cendre volante) à des taux variables compris entre 0 et $15 \%$.

L'attribution d'une note dite économique s'avère difficile car elle dépend de beaucoup de paramètres difficilement maîtrisables lors d'une phase de travaux de recherche (quantités à traiter réduites, procédés d'élaboration de matériaux non automatisés, travail à la demande...). Néanmoins, la note relative aux liants peut s'évaluer à partir des dosages, de leur prix et de leur disponibilité sur le marché. Une première estimation a conduit à retenir l'attribution des notes suivantes. Une note de 1 a été affectée pour les formulations contenant $5 \%$ de ciment, une note de 0.5 pour $5 \%$ d'incorporation de chaux et une note de 0.1 pour un ajout à hauteur de $5 \%$ de cendre volante i.e. de la Soproline ${ }^{\circledR}$. Les quantités exprimées en matières sèches et les pondérations (notes économiques) de tous les mélanges étudiés sont rassemblées dans le tableau 2.

Tableau 2. Formulations testées sur les sédiments marins.

\begin{tabular}{|c|c|c|c|c|c|}
\hline Formulation & Sédiment sec & Ciment CEM I & Soproline & Chaux vive & Note économique \\
\hline A1 & 100 & 5 & 0 & 0 & 1.0 \\
\hline A2 & 100 & 10 & 0 & 0 & 2.0 \\
\hline A3 & 100 & 12.5 & 0 & 0 & 2.5 \\
\hline A4 & 100 & 15 & 0 & 0 & 3.0 \\
\hline B1 & 100 & 15 & 0 & 10 & 4.0 \\
\hline B2 & 100 & 10 & 20 & 10 & 3.4 \\
\hline B3 & 100 & 10 & 0 & 5 & 2.5 \\
\hline B4 & 100 & 10 & 20 & 5 & 2.9 \\
\hline B5 & 100 & 20 & 0 & 10 & 5.0 \\
\hline B6 & 100 & 15 & 30 & 10 & 4.6 \\
\hline B7 & 100 & 15 & 35 & 15 & 5.2 \\
\hline B8 & 100 & 15 & 0 & 5 & 3.5 \\
\hline
\end{tabular}

Nota : Les chiffres indiquent la masse de chaque constituant en g pour $100 \mathrm{~g}$ de sédiment sec. 
Approche économique et validation de méthodes de traitements aux liants hydrauliques de sédiments marins contaminés: s2.7

Les différentes masses et pourcentages de chaque constituant y compris l'eau pour chaque gâchée ont été rapportées pour chaque mélange et chaque sédiment concerné. Ces données permettent d'obtenir des informations utiles pour expliquer les comportements mécaniques comme le ratio eau/ciment (SANNIER, 2008). Les pourcentages d'eau par gâchée sont très variables, elles dépendent du sédiment d'origine et de la chaîne de prétraitement appliquée à celui-ci. Rappelons que les dix sédiments du programme n’ont pas tous été concernés par les mêmes chaînes de traitement.

\section{Approche discriminatoire économique}

L’idée de cette approche est de tenter de discriminer des prétraitements ou procédés de traitements qui permettent à des moindres coûts de préparer les sédiments aux traitements à base de liant hydraulique et de répondre au double objectif de la méthode : solidification et stabilisation. L'indicateur pour mesurer la qualité au comportement mécanique des sédiments solidifiés est la résistance à la compression simple à 28 jours sur des éprouvettes cylindriques d'élancement 2. La stabilisation est quant à elle associée au comportement environnemental évalué à partir des essais de lixiviation (mises en solution) sur monolithe (NF X 31-211, 2000). Les résultats de ces essais tout aussi importants que les résistances mécaniques obtenues, sont disponibles dans la littérature notamment dans la série d'articles dédiée au projet SEDI.MAR.D. 83 (SANNIER, 2008 ; BENOIT-BONNEMASON et al., 2009 ; SEBY et al., 2009).

A l'issue des résultats, nous avons affecté une note "économique » aux matériaux élaborés obtenus après incorporation de liant hydraulique quels que soient le ou les prétraitements subis (SANNIER, 2008 ; SANNIER et al., 2008). Cette note globale résulte de la somme de deux notes, à savoir :

- une note en fonction de la chaîne de prétraitement et/ou traitement appliquée avant traitement par liant hydraulique qui varie de 0 (sédiment brut, aucun traitement) à 7 (sédiment dessablé déshydraté calciné). Cette pondération correspond à l'addition de la note affectée individuellement à chaque procédé. Elle est établie en fonction de la faisabilité technique et des moyens nécessaires à sa mise en œuvre (humains, matériels et consommables). Cette note est détaillée au sein du tableau 3.

- $\quad$ une note sur les formulations testées dans ce programme, établie à partir des dosages en ciment employé en prenant pour principe que plus la note est élevée, plus la quantité de ciment et d'additifs mise en œuvre est importante.

Ainsi une notation globale élevée signifie que le procédé d'élaboration s'avère onéreux, ce qui est préjudiciable dans le cadre d'une valorisation où d'autres matériaux à caractéristiques équivalentes sont sur le marché. 
Tableau 3. Pondération des traitements réalisés dans le projet SEDI.MAR.D. 83.

\begin{tabular}{|c|c|c|c|c|c|c|c|c|c|c|}
\hline Procédé & Brut & Criblage & Cor. Gran. & Dessablé & Deshydraté & COmposté & Phosphaté & CHaulé & CAlciné & \multirow[b]{3}{*}{ Note traitement } \\
\hline Note procédé & 0.0 & 0.5 & $\begin{array}{l}0.5 \\
\end{array}$ & 0.5 & 1.0 & \begin{tabular}{|l|}
1.5 \\
\end{tabular} & \begin{tabular}{|l|}
1.5 \\
\end{tabular} & 2.0 & 5.5 & \\
\hline & & & & & & & & & & \\
\hline B & $\mathrm{X}$ & & & & & & & & & 0.0 \\
\hline $\mathrm{DD}$ & $\bar{X}$ & & & $\bar{X}$ & $\bar{X}$ & & & & & 1.5 \\
\hline $\mathrm{BCO}$ & $\bar{X}$ & & & & & $\bar{X}$ & & & & 1.5 \\
\hline $\mathrm{DCO}$ & $\bar{X}$ & & & $\mathrm{X}$ & & $\bar{X}$ & & & & 2.0 \\
\hline $\mathrm{BCH}$ & $\bar{X}$ & & & & & & & $\mathrm{X}$ & & 2.0 \\
\hline BCO-CG (MA) & $\bar{X}$ & & $\mathrm{X}$ & & & $\mathrm{X}$ & & & & 2.0 \\
\hline $\mathrm{DCH}$ & $\mathrm{X}$ & & & $\mathrm{X}$ & & & & $\bar{X}$ & & 2.5 \\
\hline $\mathrm{BPCO}$ & $\bar{X}$ & & & & & $\bar{X}$ & $\bar{X}$ & & & 3.0 \\
\hline DCO-P & $\mathrm{X}$ & & & $\bar{X}$ & & $\bar{X}$ & $\mathrm{X}$ & & & 3.5 \\
\hline $\mathrm{BPCO}-\mathrm{CG}(\mathrm{MA})$ & $\mathrm{X}$ & & $\bar{X}$ & & & $\mathrm{X}$ & $\mathrm{X}$ & & & 3.5 \\
\hline DPCO & $\bar{X}$ & & & $\mathrm{X}$ & & $\bar{X}$ & $\bar{X}$ & & & 3.5 \\
\hline BCO-PD & $\bar{X}$ & $\mathrm{X}$ & & & $\bar{X}$ & $\bar{X}$ & $\bar{X}$ & & & 4.5 \\
\hline BCO-PD-CG (MA) & $\bar{X}$ & $\bar{X}$ & $\bar{X}$ & & $\bar{X}$ & $\bar{X}$ & $\bar{X}$ & & & 5.0 \\
\hline BCA & $\bar{X}$ & $\bar{X}$ & & & & & & & $\bar{X}$ & 6.0 \\
\hline DDCA & $\mathrm{X}$ & & & $\bar{X}$ & $\bar{X}$ & & & & $\bar{X}$ & 7.0 \\
\hline
\end{tabular}

Les notes finales obtenues pour chacun des traitements sont regroupées dans le tableau 4. Elles sont couplées aux résultats mécaniques obtenus, à savoir à la valeur de la résistance à la compression simple acquise à 28 jours (notée $\mathrm{Rc}_{28}$ ), et à la réponse aux tests environnementaux définissant la notion d'un matériau pouvant être valorisé en techniques routières (GTS, 2000) d'après les seuils d'acceptabilité en remblai revêtu (ADEME et al., 2006). En respectant ces critères, les produits élaborés dans ce programme ne sont pas tous valorisables comme indiqué dans la colonne de droite du tableau 4.

Tableau 4. Notes finales obtenues pour chacun des traitements appliqués dans le projet SEDI.MAR.D. 83.

Nota : * note relative au traitement ; **note relative à la formulation en liant.

\begin{tabular}{|c|c|c|c|c|c|c|}
\hline Abréviation & Formulation & Note* & Note** & Note globale & $R c_{28}(M P a)$ & Valorisable \\
\hline B & A1 & 0.0 & 1.0 & 1.0 & $\mathbf{0 . 0 7}$ & NON \\
\hline B & A2 & 0.0 & 2.0 & 2.0 & 0.15 & NON \\
\hline B & A4 & 0.0 & 3.0 & 3.0 & 0.18 & $\mathrm{NON}$ \\
\hline ВСH & A2 & 2.0 & 2.0 & 4.0 & 0.72 & \\
\hline BCH & A3 & 2.0 & 2.5 & 4.5 & 0.90 & \\
\hline BCH & A4 & 2.0 & 3.0 & 5.0 & 1.14 & OUI \\
\hline BCO & A1 & 1.5 & 1.0 & 2.5 & 0.45 & \\
\hline BCO & A2 & 1.5 & 2.0 & 3.5 & 1.01 & OUI \\
\hline BCO & A3 & 1.5 & 2.5 & 4.0 & 1.08 & OUI \\
\hline BCO & A4 & 1.5 & 3.0 & 4.5 & 1.56 & OUI \\
\hline BCO & A4 & 1.5 & 3.0 & 4.5 & 1.05 & OUI \\
\hline BCO & B1 & 1.5 & 4.0 & 5.5 & 0.82 & OUI \\
\hline BCO & B2 & 1.5 & 3.4 & 4.9 & 1.29 & OUI \\
\hline BCO & B8 & 1.5 & 3.5 & 5.0 & 1.18 & OUI \\
\hline BCO & B4 & 1.5 & 2.9 & 4.4 & 1.33 & OUI \\
\hline BCO-CG (MA) & B3 & 2.0 & 2.5 & 4.5 & 3.25 & OUI \\
\hline BCO-CG (MA) & B4 & 2.0 & 2.9 & 4.9 & 9.00 & OUI \\
\hline
\end{tabular}


Approche économique et validation de méthodes de traitements aux liants hydrauliques de sédiments marins contaminés: s2.9

\begin{tabular}{|c|c|c|c|c|c|c|}
\hline $\begin{array}{l}\text { Tableau 4. (suite, } \\
\text { Nota : * note relativ }\end{array}$ & e au traitemen & $t ; * *$ no & te relativ & e à la formula & tion en liant. & \\
\hline Abréviation & Formulation & Note* & Note** & Note globale & $R c_{28}(M P a)$ & Valorisable \\
\hline DD & A1 & 1.5 & 1.0 & 2.5 & 0.35 & NON \\
\hline DD & A2 & 1.5 & 2.0 & 3.5 & 0.80 & NON \\
\hline DD & A4 & 1.5 & 3.0 & 4.5 & 1.19 & NON \\
\hline DCH & A2 & 2.5 & 2.0 & 4.5 & 0.57 & \\
\hline DCH & A3 & 2.5 & 2.5 & 5.0 & 0.70 & \\
\hline DCH & A4 & 2.5 & 3.0 & 5.5 & 1.30 & OUI \\
\hline DCO & A1 & 2.0 & 1.0 & 3.0 & 0.37 & $\overline{\mathrm{NON}}$ \\
\hline DCO & A2 & 2.0 & 2.0 & 4.0 & 0.71 & NON \\
\hline DCO & A4 & 2.0 & 3.0 & 5.0 & 1.18 & \\
\hline DCO & B5 & 2.0 & 5.0 & 7.0 & 1.33 & OUI \\
\hline DCO & B6 & 2.0 & 4.6 & 6.6 & 1.39 & OUI \\
\hline DCO & B7 & 2.0 & 5.2 & 7.2 & 1.57 & OUI \\
\hline ВPCO & A1 & 3.0 & 1.0 & 4.0 & 0.37 & NON \\
\hline BPCO & A2 & 3.0 & 2.0 & 5.0 & 0.86 & NON \\
\hline ВPCO & A4 & 3.0 & 3.0 & 6.0 & 1.82 & \\
\hline ВPCO & A4 & 3.0 & 3.0 & 6.0 & 2.07 & OUI \\
\hline ВPCO & B1 & 3.0 & 4.0 & 7.0 & 1.61 & OUI \\
\hline BPCO & B2 & 3.0 & 3.4 & 6.4 & 2.50 & OUI \\
\hline BPCO-CG (M.A) & B3 & 3.5 & 2.5 & 6.0 & 8.50 & NON \\
\hline BPCO-CG (M.A) & B4 & 3.5 & 2.9 & 6.4 & 9.00 & NON \\
\hline DPCO & A4 & 3.5 & 3.0 & 6.5 & 2.01 & OUI \\
\hline DPCO & B1 & 3.5 & 4.0 & 7.5 & 1.61 & OUI \\
\hline DPCO & B2 & 3.5 & 3.4 & 6.9 & 2.35 & OUI \\
\hline BCA & A1 & 6.0 & 1.0 & 7.0 & 1.37 & OUI \\
\hline BCA & A2 & 6.0 & 2.0 & 8.0 & 2.61 & OUI \\
\hline BCA & A4 & 6.0 & 3.0 & 9.0 & 3.74 & OUI \\
\hline DDCA & A1 & 7.0 & 1.0 & 8.0 & 1.76 & OUI \\
\hline DDCA & A2 & 7.0 & 2.0 & 9.0 & 2.99 & OUI \\
\hline DDCA & A4 & 7.0 & 3.0 & 10.0 & 3.66 & OUI \\
\hline DCO-P & B5 & 3.5 & 5.0 & 8.5 & 1.40 & OUI \\
\hline DCO-P & B6 & 3.5 & 4.6 & 8.1 & 1.91 & OUI \\
\hline DCO-P & B7 & 3.5 & 5.2 & 8.7 & 2.01 & OUI \\
\hline BCO-PD & A4 & 4.5 & 3.0 & 7.5 & 0.90 & OUI \\
\hline BCO-PD & B1 & 4.5 & 4.0 & 8.5 & 0.84 & OUI \\
\hline BCO-PD & B2 & 4.5 & 3.4 & 7.9 & 1.22 & OUI \\
\hline BCO-PD-CG (MA) & B3 & 5.0 & 2.5 & 7.5 & 3.75 & OUI \\
\hline BCO-PD-CG (MA) & B4 & 5.0 & 2.9 & 7.9 & 9.50 & OUI \\
\hline
\end{tabular}

Les valeurs des résistances mécaniques i.e résistances à la compression simple à 28 jours $\left(\mathrm{Rc}_{28}\right)$ sont relativement faibles du fait de la nature des matériaux stabilisés, voir tableau 4. En effet, les matrices traitées représentent majoritairement des fines riches en matières organiques qui ne présentent pas de squelette minéral capable de supporter des 
contraintes élevées à la compression et où le liant est sensé apporter la cohésion à ce squelette.

\section{Visualisation et analyse des évaluations}

Une fois la pondération ou la note économique déterminée, il est possible de montrer comment évoluent les coûts de traitement en relation avec l'indicateur mécanique retenu, i.e. $\mathrm{Rc}_{28}$, et ce, pour les différents procédés de pré traitement mis en œuvre. En d'autres termes, c'est mener une étude comparative des valeurs en résistance obtenues à 28 jours pour l'ensemble des chaînes de traitement. Le tableau 5 en donne les différents symboles. L'obtention d'une valeur minimale de $1 \mathrm{MPa}$ en résistance à la compression simple constituait la valeur cible à atteindre pour une valorisation possible du point de vue mécanique en techniques routières.

Ainsi, les résultats obtenus sont regroupés sur deux figures. Le premier graphique (figure 1.) rassemble les différents procédés ou chaînes de traitement mis en œuvre sur lequel on peut identifier deux groupes aux résistances minimales (groupe $n^{\circ} 6$ ) et aux résistances maximales (groupe $n^{\circ} 5$ ).

Tableau 5. Symboles utilisés pour les prétraitements, voir figures 1 et 2.

\begin{tabular}{lllll|}
\hline $\mathrm{B}$ & $\square \mathrm{BCH}$ & $\circ \mathrm{BCO}$ & + BCO-CG (M.A. $)$ & $\triangle \mathrm{DD}$ \\
$\times \mathrm{DCH}$ & $\square \mathrm{DCO}$ & $\triangle \mathrm{BPCO}$ & + BPCO-CG (M.A) & $\bullet$ DPCO \\
$\circ \mathrm{BCA}$ & $* \mathrm{DDCA}$ & $\bullet \mathrm{DCO}-\mathrm{P}$ & $\bullet \mathrm{BCO}-\mathrm{PD}$ & + BCO-PD-CG (M.A)
\end{tabular}

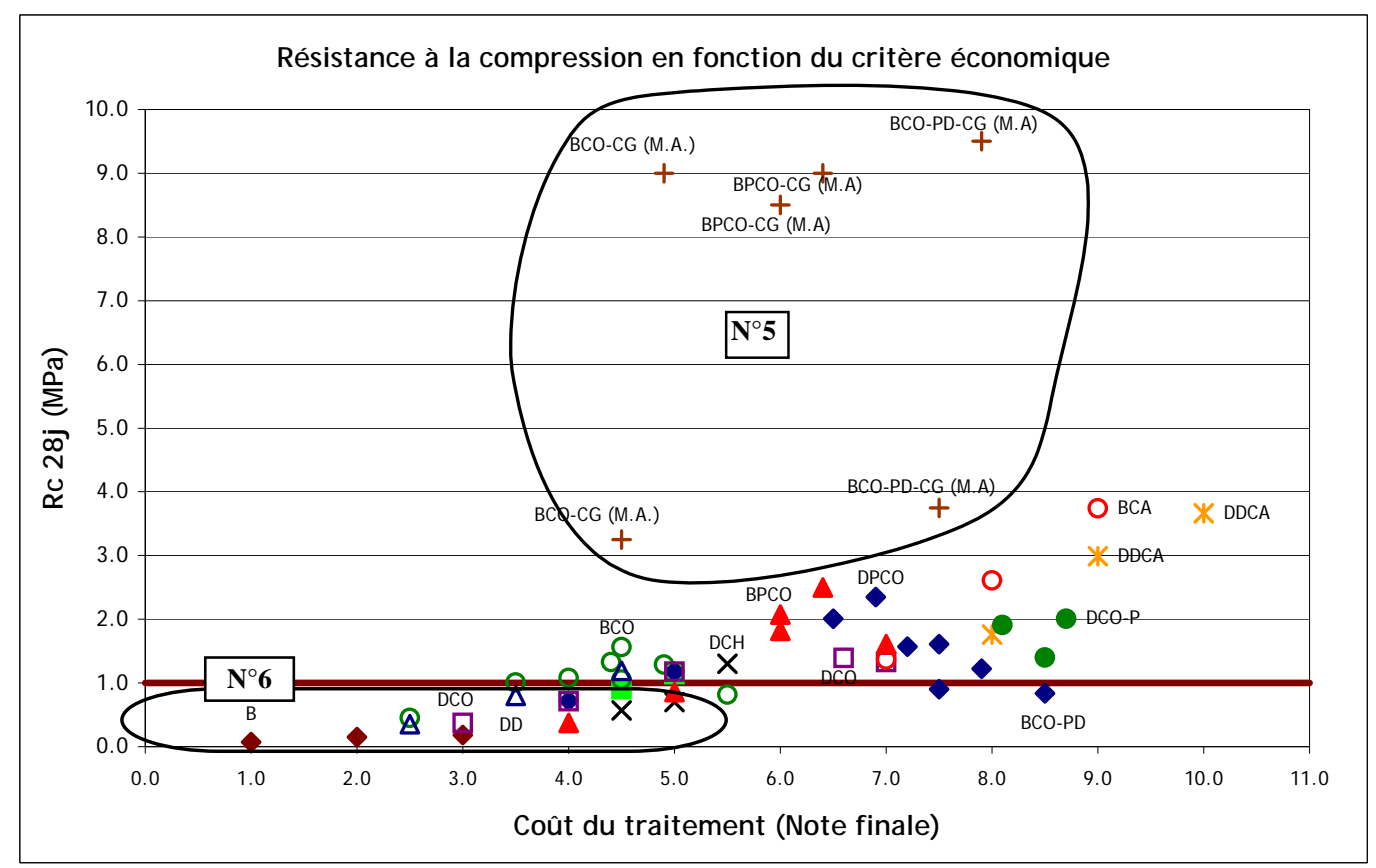

Figure 1. Relation $\mathrm{Rc}_{28}$ en fonction du coût du traitement global. 
Approche économique et validation de méthodes de traitements aux liants hydrauliques de sédiments marins contaminés: s2.11

La figure 1 montre clairement l'effet du correcteur granulométrique qui améliore le squelette granulaire et donne des résistances nettement plus élevées. Ce type de traitement correspond à la mise au point d'un matériau que l'on peut appeler « matériau alternatif ». A l'opposé, le minimum de traitement apporté aux sédiments, n'améliore peu leur résistance mécanique.

Sur la figure 2, on s'intéresse aux groupes intermédiaires. Ainsi, on ne considère plus ce type de matériau alternatif qui demande l'apport d'un matériau noble. Ceci du point de vue développement durable, est difficilement envisageable. On ne tient compte que des apports en liants proprement dit : ciment, chaux et cendre volante. L'étendue des résistances mécaniques se retrouve comprises entre 1 et $4 \mathrm{MPa}$, c'est-à-dire au dessus du seuil retenu.

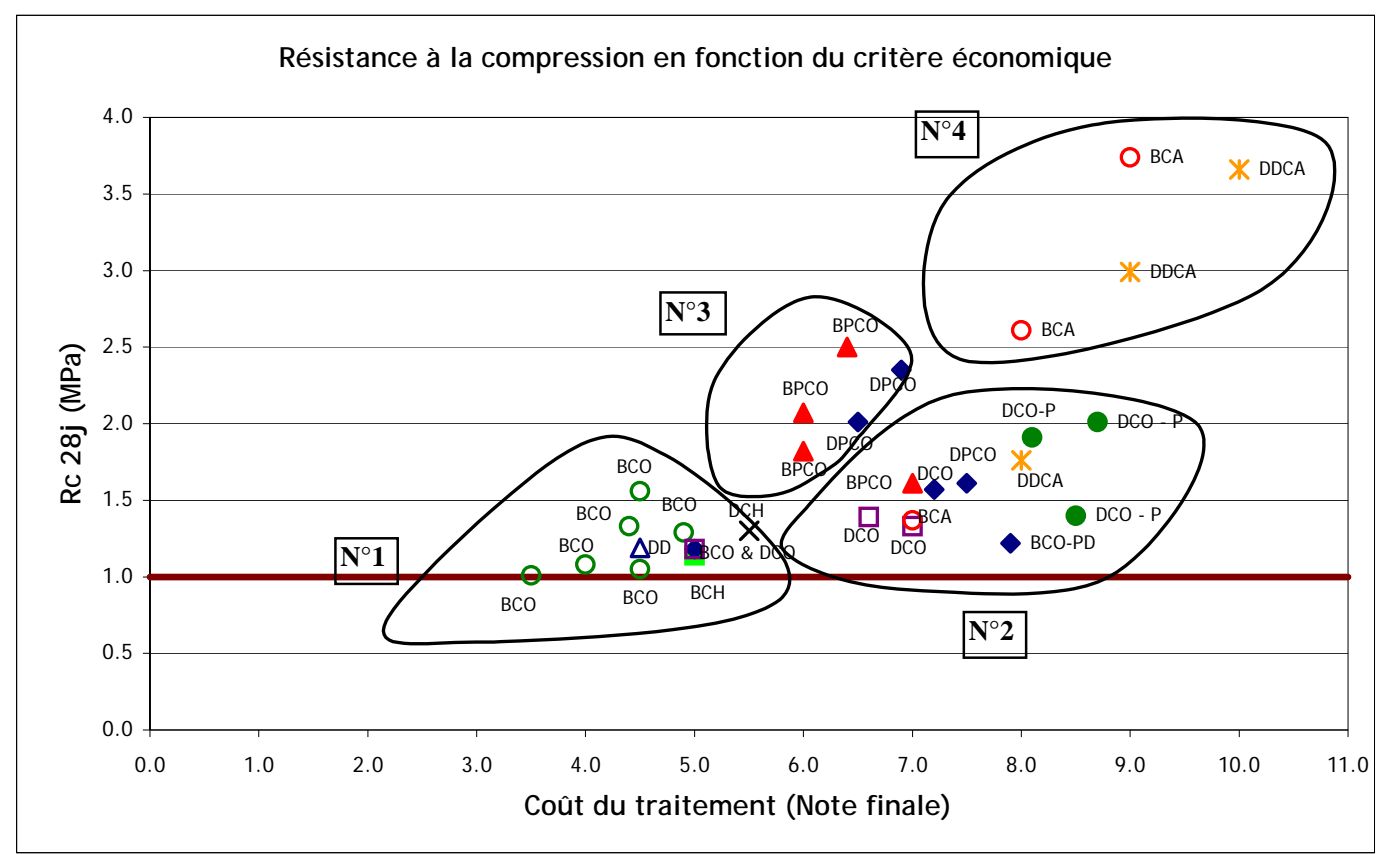

Figure 2. Relation $\mathrm{Rc}_{28}$ en fonction du coût du traitement global $\left(R c_{28} \geq a ̀ 1 \mathrm{MPa}\right.$ et sans apport granulaire.)

Des tendances observées et identifiées sur les figures 1 et 2, se dégagent six groupes de traitements et/ou familles de sédiments pré traités ou non (bruts) avec entre parenthèses l'indication du coût moyen et la valeur moyenne de la résistance mécanique obtenue:

- groupe $n^{\circ} 1$ (coût $=4.6 ; \mathrm{Rc}_{28}=1.2 \mathrm{MPa}$ ) : Il comprend les sédiments traités par compostage sur le brut et par chaulage sur les deux granulométries. Le procédé est économiquement faible mais les résistances ne sont pas nettement au-dessus du seuil. Si l'effet du chaulage n'est pas surprenant de par la connaissance de travaux antérieurs (REY, 1999), le comportement d'un sédiment composté stabilisé par un liant hydraulique n’est pas ou peu relaté dans la littérature. Ce 
procédé de prétraitement même s’il est économque, nécessite une surface au sol importante,

- groupe $\mathrm{n}^{\circ} 2$ (coût $\left.=7.6 ; \mathrm{Rc}_{28}=1.55 \mathrm{MPa}\right)$ : Il concerne des sédiments traités composés de matériaux compostés suivis d'une phosphatation et de matériaux dessablés compostés. Le bénéfice sur le comportement mécanique est très faible au vu du traitement dispendieux. La phosphatation est un procédé industriel déjà appliqué aux traitements des sols et boues polluées, mais aussi aux sédiments pollués (NGUYEN, 2006) dont les résultats sont connus. Mais il y a peut être une combinaison intéressante à organiser avec le compostage, comme il est indiqué dans le groupe 3 ,

- $\quad$ groupe $n^{\circ} 3$ (coût $=6.3 ; \mathrm{Rc}_{28}=2.1 \mathrm{MPa}$ ) : Il prend en compte tous les matériaux phosphatés suivis d'un compostage. On trouve ici un bon compromis entre les performances mécaniques et le facteur économique,

- groupe $\mathrm{n}^{\circ} 4$ (coût $=9.0 ; \mathrm{Rc}_{28}=3.3 \mathrm{MPa}$ ) : Il résulte de l'utilisation d'un procédé de calcination. Le bénéfice sur le comportement mécanique est certes important mais il s’accompagne d'un coût prohibitif. Le devenir de sédiments traités par calcination fait partie de travaux actuels (NGUYEN, 2006). Le procédé de calcination est souvent utilisé dans le cas de traitement de sédiments très contaminés, parfois associé à d'autres prétraitements,

- groupe $\mathrm{n}^{\circ 5}$ (coût $=6.2 ; \mathrm{Rc}_{28}=7.2 \mathrm{MPa}$ ) : Il comprend l'ensemble des matériaux dits alternatifs. Il semble que ce mélange soit le plus intéressant. Cependant, la seule contrepartie est la faible quantité de sédiment traité. Cette technique de correction granulométrique a été employée depuis longtemps dans la confection de matériaux élaborés. Elle a été récemment appliquée dans le cas de matériaux fins comme les sédiments (COLIN, 2003; DUBOIS, 2006 ; TRAN, 2009),

- groupe $\mathrm{n}^{\circ} 6$ (coût $\left.=4.0 ; \mathrm{Rc}_{28}=0.55 \mathrm{MPa}\right)$ : Comme l'on pouvait s'y attendre, les éprouvettes contenant les plus faibles pourcentages de ciment et sans additif présentent les plus faibles résistances à la compression. Cela concerne plus particulièrement tous les sédiments bruts et dessablés déshydratés. Ces sédiments ne sont pas appropriés à ce type de mélange sans apport d’additifs.

\section{Conclusions}

Tout d'abord, une première analyse basée sur les résultats d'un nombre important d'essais réalisés dans la phase préliminaire du programme SEDI.MAR.D.83, (SANNIER, 2008), avait permis de :

- de confirmer la faisabilité technique de ce type de traitement à l'aide de liants hydrauliques,

- d’identifier les pourcentages optimaux de ciment et les éventuels additifs dont l'ajout constitue un apport intéressant à mettre en œuvre, 
Approche économique et validation de méthodes de traitements aux liants hydrauliques de sédiments marins contaminés: s2.13

- de valider la faisabilité environnementale par rapport aux filières de valorisation envisageables,

- de définir les coupures granulométriques appropriées, (sédiments dessablés).

S'en est suivie, une campagne complète d'essais de comportement mécanique et environnemental sur un ensemble de sédiments marins bruts ou prétraités stabilisés et/ou solidifiés à l'aide de liants hydrauliques. L'étude, présentée dans cette note, s'est focalisée sur les essais de comportement mécaniques tout en mettant en avant la notion de matériau valorisable (tableau 4) en introduisant la notion de critère économique qui fait souvent défaut dans de nombreux travaux de recherche sur la valorisation de sédiments marins. Ainsi une méthodologie a été mise en œuvre qui a permis d'identifier les procédés les plus efficaces à un traitement par liants hydrauliques.

Quelle que soit la chaîne de prétraitement appliquée aux sédiments marins, le traitement par liants a montré que la stabilisation et la solidification obtenues peuvent être suffisantes dans le cas d'une valorisation de type matériau à destination des techniques routières et remblaiement (sous-couches routières et remblais revêtus). En effet, les valeurs de résistance à la compression sont suffisantes et que les résultats des essais environnementaux comparés aux seuils d'acceptabilité fixé par le guide du ministère de l'Equipement (ADEME et al., 2006) s'avèrent satisfaisants (BENOIT-BONNEMASON et al., 2009 ; SANNIER, 2008 ; SEBY et al., 2009).

Cette approche économique, simple certes, a permis d'identifier des groupes de traitements à performances équivalentes associant la notion de coût, de résistance mécanique et de produit valorisable. Les points forts de cette étude sont les suivants :

- Le procédé de traitement par phosphatation accompagné d'un compostage apporte le meilleur compromis résistance/coût pour un traitement au liant hydraulique. Ceci correspond au groupe 3 .

- L'incorporation d'additifs à moindre coût comme les cendres volantes i.e. la Soproline ${ }^{\circledR}$ ou la chaux vive peut s’avérer intéressante, notamment en substitution au ciment. De plus ces additifs jouent un rôle sur la teneur en eau, la teneur en matières organiques, sur la résistance à long terme. L'effet pouzzolanique n’est pas considéré ici car des essais au-delà de 28 jours sont nécessaires (LEVACHER et al., 2008 ; SILITONGA et al., 2009). De par ces rôles, les chaînes de prétraitement peuvent être réduites.

- Une solution alternative aux traitements par phosphatation et/ou calcination qui restent très onéreux repose sur l'utilisation d'une méthode de traitement avec correcteur granulométrique. Bien entendu, cela passe par la valorisation de plus petits volumes de sédiments (dosage à faibles pourcentages de sédiments marins) en ajoutant un matériau de carrière ou équivalent. En effet, de par son apport d'éléments plus grossiers, il réduit les concentrations en agents inhibiteurs à la prise du liant et augmente la cohésion du squelette minéral. 


\section{Références bibliographiques}

ADEME, BRGM, CETE, INERIS, LCPC, MEDD, METLTM, SETRA (2006). Acceptabilité des déchets et des matériaux en techniques routières, Guide technique, Rapport ADEME, pp 66-82.

AQUA J.-L., BOISSERY P., ALZIEU C. (2009). Un programme opérationnel d'identification des modes de gestion durable des sédiments marins : le projet SEDI.MAR.D. 83, Revue Paralia, ${ }^{\circ}$ 2, pp s1.1-s1.12.

BENOIT-BONNEMASON C., SEBY F., TURLOT J.-C., JOURDAN M., ALZIEU C., AQUA J.-L., SANNIER L., DONARD O.F.-X. (2009). Analyse statistique des données obtenues sur les sédiments traités au site pilote de SEDI.MAR.D. 83 Revue Paralia, in press.

BOUTOUIL M. (1998). Traitement des vases de dragage par solidification/stabilisation à base de ciments et additifs, Thèse Université du Havre, $278 \mathrm{p}$.

COLIN D. (2003). Valorisation de sédiments fins de dragage en technique routière, Thèse Université de Caen, 180 p.

DUAN Z. (2008). Caractérisation, stabilisation et solidification de sédiments marins, Thèse Université de Caen, $144 \mathrm{p}$.

DUBOIS V. (2006). Etude du comportement physico mécanique et caractérisation environnementale des sédiments marins - Valorisation en technique routière, Thèse Ecole Nationale Supérieure des Mines, Douai, 190 p et annexes 95 p.

GROSDEMANGE D., LEVEQUE F., DROUSIE D., AQUA J-L., MEHU J., BAZIN C. (2008). The SEDI.MAR.D. project: presentation and results. International Symposium on Sediment Management -I2SM, Lille, pp 181-186.

GTS (2000). Traitement des sols à la chaux et aux liants hydrauliques - Guide technique - Edition LCPC-SETRA, 240 p.

JOCE (2003). Décision n²003/33/CE du 19 décembre2002 établissant des critères et des procédures d'admission des déchets dans les décharges, conformément à l'article 16 et à l'annexe II de la directive 1999/31/CE, JOCE nº11 du 16 janvier 2003, 27 p.

LEVACHER D. (2008). Caractérisation géotechnique des sédiments- L'exemple des ports méditerranéens, Colloque Gestion durable des sédiments, Monaco 28-29 octobre 2008, diaporama, in press.

LEVACHER D., DUAN Z., LIU Z., SANCHEZ M. (2008a). Caractérisation mécanique de sédiments de ports méditerranéens en vue de leur réutilisation en remblai, Rapport d'avancement $\mathrm{N}^{\circ}$ 1, Réf. $\mathrm{N}^{\circ}$ CFL-07-CG83-01, Centre Français du Littoral, 64 p.

LEVACHER D., DUAN Z., SANCHEZ M. (2008b). Caractérisation mécanique de sédiments de ports méditerranéens stabilisés, Rapport d'avancement $\mathrm{N}^{\circ} 2$, Réf. No CFL-07-CG83-02, Centre Français du Littoral, 50 p. 
Approche économique et validation de méthodes de traitements aux liants hydrauliques de sédiments marins contaminés: s2.15

LEVACHER D., LIANG Y. (2008c). Evolution des propriétés physico-chimiques et amélioration de la résistance de sédiments de ports méditerranéens par des procédés de solidification, Rapport d'avancement $N^{\circ} 3$, Réf. N CFL-07-CG83-03, Centre Français du Littoral, 79 p.

LEVACHER D., SANCHEZ M., DUAN Z., LIANG Y. (2009). Caractérisation géotechnique de sédiments méditerranéens pour une valorisation, Revue Paralia, in press.

NF X 31- 211 (2000). Déchets - Essais de lixiviation d'un déchet solide initialement massif ou généré par un procédé de solidification.

NGUYEN T. B. (2008). Valorisation des sédiments de dragage traités par le procédé dans des matériaux d'assises de chaussée - comportement mécanique et environnemental, Thèse Institut National des Sciences Appliquées, Toulouse, 244 p.

PETAVY F., RUBAN V., CONIL P. (2007). Mise en place d'une unité pilote de traitement des sédiments issus de l'assainissement pluvial, Revue La Houille Blanche, $\mathrm{N}^{\circ}$ 05-2007, pp 113-119.

PETAVY F., RUBAN V., CONIL P., VIAU J.-Y. (2008). Reduction of sediment micropollution by jeans of a pilot plant, Water Science Technology Journal, WST 57-10, pp 1611-1617.

REY F. (1999). Etude physico - mécanique et environnementale du composite vasechaux- additifs, Thèse Université de Caen, 272 p.

SANNIER L. (2008). Limites et performances des caractéristiques mécaniques et environnementales de sédiments marins contaminés traités par liant hydraulique. Application au pilote SEDI.MAR.D. 83, Mémoire de Master de Recherche, Université de Caen, 106 p.

SANNIER L., LEVACHER D., JOURDAN M. (2008). Discrimination économique de méthodes de traitements de sédiments marins contaminés traités à l'aide de liants hydrauliques, Journées Nationales Génie Côtier - Génie Civil, Sophia-Antipolis, pp 821-830. Disponible en ligne [URL http://www.paralia.fr/jngcgc/10_77_sannier.pdf] SEBY F., BENOIT-BONNEMASON C., TESSIER E., ALZIEU C., AQUA J.-L., SANNIER L., DONARD O.F.-X. (2009). Etude de l'évolution des formes chimiques des métaux dans des sédiments marins dragués stockés à terre, Revue Paralia, in press.

SEMCHA A. (2006). Valorisation des sédiments de dragage - application dans le BTP. Cas de barrages algériens, Thèse Université de Reims Champagne Ardenne, 173 p.

SILITONGA E., LEVACHER D., MEZAZIGH S. (2009). Effects of the use of fly ash as a binder on the mechanical behaviour of treated dredged sediments, Environment Technology Review, Vol. 30, n 8 , pp 798-807.

TRAN N.T. (2009). Valorisation de sédiments marins et fluviaux en technique routière, Thèse Ecole Nationale Supérieure des Mines, Douai, 187 p. 
s2.16 : Revue Paralia - n 2 (2009) 